\title{
Application of blood cadmium determination to industry using a punched disc technique
}

\author{
A. A. CERNIK and M. H. P. SAYERS \\ Central Reference Laboratory. Department of Employment, London W2 ${ }^{1}$
}

\begin{abstract}
Cernik, A. A. and Sayers, M. H. P. (1975). British Journal of Industrial Medicine, 32, 155-162. Application of blood cadmium determination to industry using a punched disc technique. A paper disc flameless atomic absorption spectroscopy (AAS) method is described for the determination of cadmium (Cd) in blood, enabling difficulties in sample preparation to be minimized. By control of the ashing step the matrix can be removed without loss of cadmium. Problems with the fast signal response during atomization can be met by spectral band width and temperature control. At the $106 \mathrm{pg}$ level $(471 \mathrm{nmol} \mathrm{Cd} / 1$ blood; $5.3 \mu \mathrm{g} / 100 \mathrm{ml})$ the relative standard deviation (RSD) was $\mathbf{0 . 0 6}$. Results in four industrial situations are reported. This description of the method should facilitate further investigation of its application to industry using capillary or venous blood.
\end{abstract}

The increasing interest in environmental pollutants has focused recent attention on cadmium (Cd). This metal is widely used in industry but knowledge regarding its absorption and excretion is incomplete, thus rendering difficult the interpretation of industrial exposure using biological methods. Measured by cathode ray polarography (POL), cadmium in the urine of the general population is less than 44.5 $\mathrm{nmol} / \mathrm{l}(5 \mu \mathrm{g} / \mathrm{l})$ in our experience. In the industrially exposed greater quantities may be found. Urinary excretion of cadmium is commonly used as an index of absorption, and the finding of $222 \mathrm{nmol} / \mathrm{l}(25$ $\mu \mathrm{g} / \mathrm{l})$ is considered evidence of undue exposure. This does not, however, reflect the total body burden or the concentration in vulnerable organs such as the kidney or the lung.

Absorbed cadmium is conveyed in the bloodstream, either cell or plasma bound, throughout the body. Correlation between the cadmium urine (CdU) and blood $(\mathrm{CdB})$ is poor, and indeed neither measurement is regarded as a good index of absorption by some authorities. Moreover the CdU does not necessarily reflect recent exposure. Tissue biopsy has been advocated by Ullucci and Hwang (1973)

${ }^{1}$ Now the medical laboratory 0. the Health and Safety Executive, Baynards House, London W2 in view of the problems of blood and urinary findings.

From the practical viewpoint a simple test is needed for the detection of evidence of undue absorption of $\mathrm{Cd}$ in industrial workers which is acceptable to workers and management and which can be related to exposure. It should alert the industrial hygienist to advise on control of the environment before a concentration in the critical organs can occur which might result for example in tubular nephritis with associated proteinuria.

This paper describes recent developments in the use of atomic absorption spectroscopy (AAS) for the estimation of $\mathrm{CdB}$ which can be readily adopted for the study of venous or capillary specimens collected from workers in the factory.

Comparative results using capillary blood are included in order to encourage the use of this method of sampling which may prove more acceptable for industrial workers.

Among recent AAS techniques the use of the tantalum sampling boat has achieved a relative detection limit for cadmium of $1.8 \mathrm{nmol} / 1(0.2 \mathrm{ng} / \mathrm{ml})$ using a $0.5 \mathrm{ml}$ sample of blood, but 16 hours were needed for oxidation (Hauser, Hinners, and Kent, 1972). Ediger and Coleman (1973) ignited $10 \mathrm{ml}$ of 
blood in a Delves sampling cup near the flame and obtained results comparable to those using an extraction/graphite furnace technique. There may be loss of cadmium using this method of oxidation since the vapour pressure temperature (VPT) of cadmium is little higher than that of burning blood. Other methods using the Delves cup after pre-treatment of the sample have shown that recoveries, reproducibility, and sensitivity are satisfactory (Joselow and Bogden, 1972; Lieberman, 1973). The latter also reported that the method overcame the interference effects of different matrices. Ullucci and Hwang (1973) favoured solvent extraction of the cadmium prior to determination on a tantalum ribbon but calibrated the blood figures with aqueous standards.

There are few published references to flameless methods. Dolinsek, Stupar, and Glazer (1972) determined cadmium in blood using the graphite furnace without pre-treatment of the sample which was dried, ashed, and atomized in situ. This direct approach to analysis of metals has the advantage of minimizing problems due to contamination. The graphite furnace technique enables cadmium to be determined on capillary or venous samples directly without pre-treatment with adequate sensitivity.

\section{Materials and methods}

\section{Collection of specimens}

Blood Venous or capillary blood samples were collected as described by Cernik and Sayers (1971).

Urine Casual specimens of urine were collected from workers in the factory and examined for cadmium by POL. Corrections were not made for specific gravity (SG), which varied widely producing spurious results where it deviated appreciably from 1016 as pointed out by Rainsford (1968). The observed SGs are given in Tables 7 and 8.

\section{Apparatus \\ A Varian Techtron AA5 spectrometer was used with a CR63 graphite furnace workhead. The graphite cups, $4.5 \mathrm{~mm}$ in diameter, were supported by two side electrodes with the correct end radius ${ }^{1}$. Other features were as described by Cernik (1974) except for the following: cadmium lamp current $4 \mathrm{~mA}$; spectral band width (SBW) $0.33 \mathrm{~nm}$; wavelength $228.8 \mathrm{~nm}$. \\ Workhead power controls For ashing, 12 seconds at $300^{\circ} \mathrm{C}$ $( \pm 10)$; for atomization, 2.0 seconds at $600^{\circ} \mathrm{C}( \pm 10)$ (arbitrary control units 5.6 and 3.2 respectively).}

\section{Method}

All blood specimens whether venous or capillary examined by AAS were done by the punched disc technique using $4.0 \mathrm{~mm}$ discs after previous spotting in the case of venous blood (Cernik, 1974). Non-atomic absorption that appeared in the cadmium position was controlled to 0.01 absorbance by the use of a hydrogen lamp. A non-interfering second peak was ignored (Fig. 1).

Working standards Cadmium chloride $\left(\mathrm{CdCl}_{2} 2.5 \mathrm{H}_{2} \mathrm{O}\right)$

${ }^{1}$ Both obtainable from MCP Electronics, Alperton, Wembley, Middlesex HA0 4PE

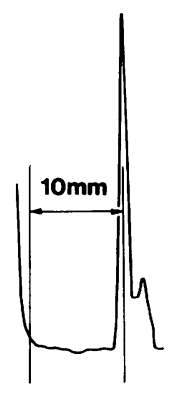

ASHING 5.6-12secs.

ATOMIZATION 3.2-2.0secs.

$\left(600^{\circ} \mathrm{C}\right)$

4.0mm Cd discs

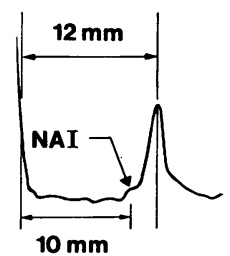

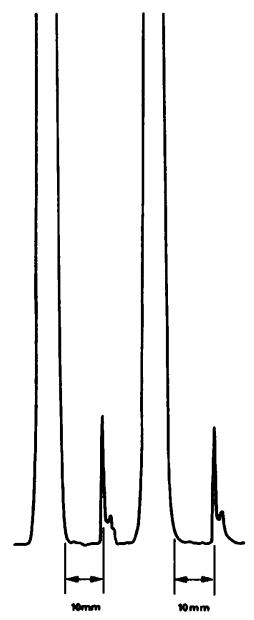

Cd LAMP

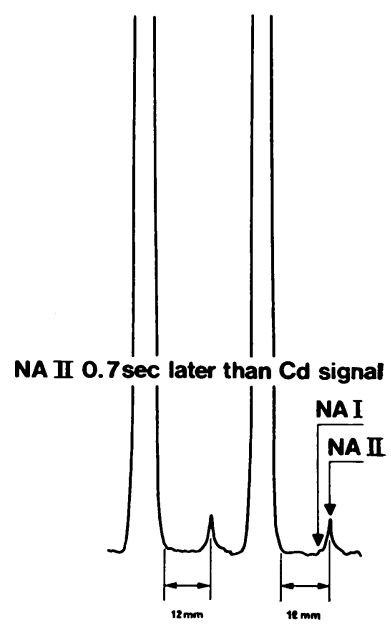

H2 LAMP
FIG. 1. Non-atomic signal found in the cadmium position when $4.0 \mathrm{~mm}$ blood discs are atomized at $600^{\circ} \mathrm{C}$ (NA $=$ non-atomic).

solution was mixed with blood samples to give a final added concentration of $178,356,534,712$ and 890 $\mathrm{nmol} \mathrm{Cd} / 1$ blood $(0 \cdot 0,2 \cdot 0,4 \cdot 0,6 \cdot 0,8 \cdot 0$, and $10 \cdot 0 \mu \mathrm{g} / 100$ $\mathrm{ml})$.

Ashing of matrix Precise control of the voltage applied over a given time constant is important for the ashing of the matrix because of the volatility of cadmium. Previous work with lead using the same technique had shown that with the voltage required to achieve $300^{\circ} \mathrm{C}$ for 12 seconds complete removal of all the molecular absorption from the matrix resulted. The same ashing system was necessary for cadmium because the matrix was identical, but unlike lead there was only approximately $100^{\circ} \mathrm{C}$ difference between the ashing temperature and the VPT of cadmium. The possibility that cadmium might vapourize at lower temperatures than its VPT was considered, and to check this discs of blood punched from one sample were ashed at increasing voltage setting and the cadmium content was determined (Table 1).

Atomization and spectral band width effect Using a relatively high atomization temperature $\left(1200^{\circ} \mathrm{C}\right)$ calibration graphs obtained from the range $0-890 \mathrm{nmol} \mathrm{Cd} / \mathbf{l}$ blood $(0-10 \mu \mathrm{g} / 100 \mathrm{ml})$ curved irrespective of spectral band width (SBW) $(0.15$ to $0.99 \mathrm{~nm})$. Use of SBW 
TA B LE 1

LosS OF CADMIUM BY INCREASING THE Temperature at the Matrix Ashing Stage

\begin{tabular}{l|c|c}
\hline $\begin{array}{c}\text { Ash control } \\
\text { setting }\end{array}$ & $\begin{array}{c}\text { Temperature } \\
\left({ }^{\circ} \mathrm{C} \pm 10\right)\end{array}$ & $\begin{array}{c}\% \text { Loss } \\
\text { cadmium }\end{array}$ \\
\hline 5.6 & 300 & $\mathrm{Nil}$ \\
$5 \cdot 7$ \\
$5 \cdot 8\{$ All at 12 & 340 & $\mathrm{Nil}$ \\
5.9 \\
6.1
\end{tabular}

narrower than $0 \cdot 15 \mathrm{~nm}$ resulted in unacceptable signal/ noise ratios. Reproducibility was also erratic. Reduction of the volatilization temperature to approximately $600^{\circ} \mathrm{C}$ produced linear analytical graphs optimumly at an SBW of $0.33 \mathrm{~nm}$ (Fig. 2). To establish the optimum lowest temperature setting for the best vapourization of the cadmium, blood discs from a sample were atomized at increasing temperatures (using SBW of $0.33 \mathrm{~nm}$ ) and the residual cadmium was checked (Table 2 ).

\section{Results}

Venous and capillary blood specimens and urine

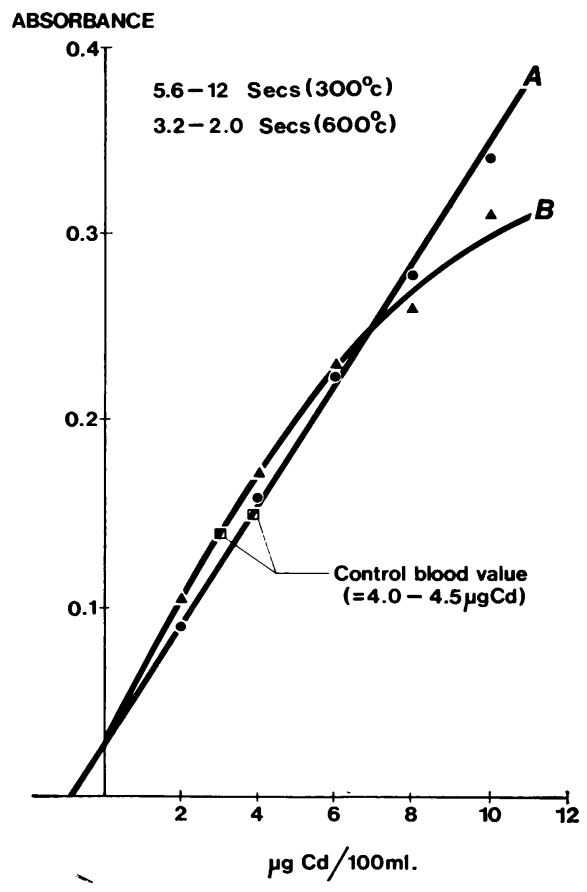

FIG. 2. Effect of slit width and volatilization temperature on the linearity of calibration. A, spectral band width $0.33 \mathrm{~nm}$; B, spectral band width $0.99 \mathrm{~nm}$.

Conversion: Traditional Units to SI.

-Cadmium: $1 \mu \mathrm{g} / 100 \mathrm{ml}=88.9 \mathrm{nmol} / 1$.
TA B LE 2

Atomization Temperature and Residual CADMIUM (SBW 0.33 nm)

\begin{tabular}{c|c|c|c}
\hline $\begin{array}{c}\text { Atomize } \\
\text { control } \\
\text { setting } \\
(\text { all } 2.0 \mathrm{sec})\end{array}$ & $\begin{array}{c}\text { Temperature } \\
\left({ }^{\circ} \mathrm{C} \pm 10^{\circ}\right)\end{array}$ & $\begin{array}{c}\text { Cadmium } \\
\text { peak } \\
\text { height }\end{array}$ & $\begin{array}{c}\text { Residual } \\
\text { cadmium } \\
\text { peak } \\
\text { height }\end{array}$ \\
\hline-1.0 & 450 & 32 & 8.0 \\
2.5 & 490 & 35 & $7 \cdot 0$ \\
3.0 & 515 & 35 & 3.0 \\
3.2 & 600 & 39 & 1.0 \\
3.5 & 625 & 43 & 1.0 \\
\hline
\end{tabular}

samples were collected from workers engaged in two industrial processes with substantial risk using cadmium pigment manufacture and smelting respectively. The first is a dusty process where ingestion is the chief mode of absorption whereas fume is the principal hazard in the second. Specimens were also collected from workers where there was little cadmium risk such as brazing and dry battery manufacture and from non-exposed controls.

\section{Precision and accuracy}

Three different naturally occurring cadmium concentrations in blood were determined, and the results from 11 replications are shown in Table 3. To evaluate the punched disc (PD) results we compared both the capillary and venous samples against results obtained using our standard method of cathode ray polarography in 14 cases (Table 4). The correlation between the venous results done by PD and POL was 0.81 and between the capillary results and POL was 0.85 . The corresponding correlation between the 14 capillary and venous results was 0.96 .

\section{Normal values}

Ten volunteers with no history of cadmium exposure were sampled by capillary and venous methods and

TABLE 3

Precision of Replicate Analyses using $4.0 \mathrm{~mm}$ Discs at Three Different Concentrations of Blood CaDMIUM

\begin{tabular}{cc|cc|c}
\hline \multicolumn{2}{c|}{ Mean } & Standard deviation & $\begin{array}{c}\text { Relative } \\
\text { standard } \\
\text { deviation }\end{array}$ \\
\cline { 1 - 2 }$\mu \mathrm{g} / 100 \mathrm{ml}$ & nmol/l & & & 0.088 \\
\cline { 1 - 2 } 0.8 & 71 & 0.07 & $(6 \cdot 2)$ & 0.055 \\
2.0 & 178 & 0.11 & $(9 \cdot 8)$ & 0.043 \\
8.2 & 729 & 0.35 & $(31 \cdot 1)$ & \\
\hline
\end{tabular}

No. of replications at each concentration $=11$ 
TABLE 4

Comparison of Micromethod and Cathode Ray Polarography $(\mu \mathrm{g} \mathrm{Cd} / 100 \mathrm{ml}$ blood)

\begin{tabular}{|c|c|c|c|c|c|c|}
\hline \multirow[b]{3}{*}{$\begin{array}{r}1 \\
2 \\
3 \\
4 \\
5 \\
6 \\
7 \\
8 \\
9 \\
10 \\
11 \\
12 \\
13 \\
14\end{array}$} & \multicolumn{4}{|c|}{$4.0 \mathrm{~mm} P D$} & \multirow{2}{*}{\multicolumn{2}{|c|}{$\frac{\text { Polarography }}{\text { Venous }}$}} \\
\hline & \multicolumn{2}{|c|}{ Venous } & \multicolumn{2}{|c|}{ Capillary } & & \\
\hline & $\begin{array}{l}0.4 \\
0.4 \\
0.5 \\
0 \cdot 5 \\
0.9 \\
1 \cdot 4 \\
2 \cdot 2 \\
2 \cdot 8 \\
4 \cdot 8 \\
5 \cdot 0 \\
5 \cdot 7 \\
6.5 \\
6.8 \\
7 \cdot 8\end{array}$ & $\begin{array}{l}(35 \cdot 6)^{1} \\
(35 \cdot 6) \\
(44 \cdot 5) \\
(44 \cdot 5) \\
(80 \cdot 1) \\
(125) \\
(196) \\
(249) \\
(427) \\
(445) \\
(507) \\
(578) \\
(605) \\
(694)\end{array}$ & $\begin{array}{l}0 \cdot 6 \\
0 \cdot 6 \\
1 \cdot 2 \\
1 \cdot 1 \\
1 \cdot 4 \\
1 \cdot 4 \\
1.9 \\
5 \cdot 4 \\
5 \cdot 5 \\
4 \cdot 8 \\
5 \cdot 0 \\
7 \cdot 4 \\
7 \cdot 2 \\
7 \cdot 7\end{array}$ & $\begin{array}{c}(53 \cdot 4)^{1} \\
(53 \cdot 4) \\
(107) \\
(97 \cdot 9) \\
(125) \\
(125) \\
(169) \\
(480) \\
(489) \\
(427) \\
(445) \\
(658) \\
(641) \\
(685)\end{array}$ & $\begin{array}{l}0.5 \\
0.9 \\
0.8 \\
0.8 \\
1.6 \\
1.0 \\
3.0 \\
3.5 \\
8.1 \\
4.9 \\
1.9 \\
5.4 \\
5.5 \\
6.0\end{array}$ & $\begin{array}{c}(44 \cdot 5)^{1} \\
(80 \cdot 1) \\
(71 \cdot 2) \\
(71 \cdot 2) \\
(142) \\
(89) \\
(267) \\
(311) \\
(721) \\
(436) \\
(169) \\
(480) \\
(489) \\
(534)\end{array}$ \\
\hline $\begin{array}{c}\text { Mean } \\
\text { SD }\end{array}$ & $\begin{array}{l}3 \cdot 26 \\
2 \cdot 73\end{array}$ & $\begin{array}{l}(290) \\
(243)\end{array}$ & $\begin{array}{l}3 \cdot 66 \\
2 \cdot 73\end{array}$ & $\begin{array}{l}(326) \\
(243)\end{array}$ & $\begin{array}{l}3 \cdot 14 \\
2 \cdot 45\end{array}$ & $\begin{array}{l}(279) \\
(218)\end{array}$ \\
\hline
\end{tabular}

${ }^{1}$ Corresponding SI units (nmol/l) in parentheses

the results were compared (Table 5). Findings in a further seven capillary and 16 venous samples are also shown. The range of all results was $0 \cdot 89-71 \cdot 2$ $\mathrm{nmol} / 1(0.01-0.8 \mu \mathrm{g} / 100 \mathrm{ml})$ with a mean of 26.7 $(0 \cdot 30)$.
Workers engaged in brazing, with no exposure for 5-8 weeks

Thirty-three workers in a Naval dockyard who had been engaged in pipe jointing by silver brazing with cadmium-containing alloy were examined. None of

TABLE 5

Blood Cadmium in the UneXPosed $(\mu \mathrm{g} / 100 \mathrm{ml}$ blood $)$

\begin{tabular}{|c|c|c|c|c|c|c|c|}
\hline & \multicolumn{2}{|c|}{ Venous } & \multicolumn{2}{|c|}{ Capillary } & & \multicolumn{2}{|c|}{ Venous } \\
\hline $\begin{array}{r}1 \\
2 \\
3 \\
4 \\
5 \\
6 \\
7 \\
8 \\
9 \\
10 \\
11 \\
12 \\
13 \\
14 \\
15 \\
16 \\
17\end{array}$ & $\begin{array}{l}0 \cdot 2 \\
0.2 \\
0.2 \\
0 \cdot 8 \\
0 \cdot 5 \\
0 \cdot 2 \\
0 \cdot 2 \\
0 \cdot 8 \\
0 \cdot 3 \\
0 \cdot 6\end{array}$ & $\begin{array}{l}(17 \cdot 8)^{1} \\
(17 \cdot 8) \\
(17 \cdot 8) \\
(71 \cdot 2) \\
(44 \cdot 5) \\
(17 \cdot 8) \\
(17 \cdot 8) \\
(71 \cdot 2) \\
(26 \cdot 7) \\
(53 \cdot 4)\end{array}$ & $\begin{array}{l}0 \cdot 2 \\
0 \cdot 2 \\
0 \cdot 2 \\
0 \cdot 6^{*} \\
0 \cdot 5 \\
0 \cdot 3 \\
0 \cdot 2 \\
0 \cdot 6^{*} \\
0 \cdot 2 \\
0 \cdot 6^{*} \\
0 \cdot 3^{*} \\
0 \cdot 6^{*} \\
0 \cdot 14 \\
0 \cdot 4^{*} \\
0 \cdot 16 \\
0 \cdot 5^{*} \\
0 \cdot 15\end{array}$ & $\begin{array}{l}(17 \cdot 8)^{1} \\
(17 \cdot 8) \\
(17 \cdot 8) \\
(53 \cdot 4) \\
(44 \cdot 5) \\
(26 \cdot 7) \\
(17 \cdot 8) \\
(53 \cdot 4) \\
(17 \cdot 8) \\
(53 \cdot 4) \\
(26 \cdot 7) \\
(53 \cdot 4) \\
(12 \cdot 5) \\
(35 \cdot 6) \\
(14 \cdot 2) \\
(44 \cdot 5) \\
(13 \cdot 3)\end{array}$ & $\begin{array}{l}1 \\
2 \\
3 \\
4 \\
5 \\
6 \\
7 \\
8 \\
9 \\
10 \\
11 \\
12 \\
13 \\
14 \\
15 \\
16 \\
\text { (Smokin }\end{array}$ & $\begin{array}{l}0 \cdot 15 \\
0 \cdot 02 \\
0 \cdot 01 \\
0 \cdot 15 \\
0 \cdot 3 \\
0 \cdot 3 \\
0 \cdot 4 \\
0 \cdot 03 \\
0 \cdot 10 \\
0 \cdot 06 \\
0 \cdot 11 \\
0 \cdot 4 \\
0 \cdot 3 \\
0 \cdot 3 \\
0 \cdot 3 \\
0 \cdot 2 \\
\text { history }\end{array}$ & $\begin{array}{l}(13.3)^{1} \\
(1 \cdot 8) \\
(0.89) \\
(13.3) \\
(26.7) \\
(26.7) \\
(35.6) \\
(2 \cdot 7) \\
(8 \cdot 9) \\
(5 \cdot 3) \\
(9 \cdot 8) \\
(35.6) \\
(26.7) \\
(26.7) \\
(26.7) \\
(17.8) \\
\text { unknown) }\end{array}$ \\
\hline $\begin{array}{l}\text { Mean } \\
\text { Range } \\
\text { SD }\end{array}$ & $\begin{array}{c}0.4 \\
0.2-0.8 \\
0.24\end{array}$ & $\begin{array}{l}(35 \cdot 6) \\
(17 \cdot 8-71 \cdot 2) \\
(21 \cdot 4)\end{array}$ & $\begin{array}{c}0.68 \\
0 \cdot 14-0.6 \\
0 \cdot 17\end{array}$ & $\begin{array}{l}(60 \cdot 5) \\
(12 \cdot 5-53 \cdot 4) \\
(15 \cdot 1)\end{array}$ & & $\begin{array}{r}0 \cdot 19 \\
1-0 \cdot 40 \\
0 \cdot 10\end{array}$ & $\begin{array}{l}(16.9) \\
(0.9-35 \cdot 6) \\
(8 \cdot 9)\end{array}$ \\
\hline
\end{tabular}

${ }^{1}$ Corresponding SI units (nmol/l) in parentheses 
TABLE 6

Blood Cadmium in Exposed and Unexposed Populations $(\mu \mathrm{g} / 100 \mathrm{ml}$ blood $)$

\begin{tabular}{|c|c|c|c|c|c|c|c|c|}
\hline \multicolumn{3}{|c|}{ Laboratory } & $\begin{array}{l}\text { Unexposed } \\
\text { staff }\end{array}$ & $\begin{array}{c}\text { Naval } \\
\text { dockyard } \\
\text { (silver } \\
\text { brazing) }\end{array}$ & $\begin{array}{c}\text { Battery } \\
\text { manufacture }\end{array}$ & $\begin{array}{l}\text { Pigment } \\
\text { factory } \\
(\text { dust } \\
\text { exposure })\end{array}$ & $\begin{array}{l}\text { Smelting } \\
\text { factory- } \\
\text { (fume } \\
\text { exposure) }\end{array}$ & $\begin{array}{l}\text { Smelting } \\
\text { factory-2 } \\
\text { (fume } \\
\text { exposure) }\end{array}$ \\
\hline No. of work & & . & 10 & 33 & 130 & 14 & 17 & 8 \\
\hline $\begin{array}{l}\text { Mean } \\
\text { Venous } \\
\text { Capillary }\end{array}$ & $\begin{array}{l}\cdots \\
\cdots\end{array}$ & $\begin{array}{l}\cdots \\
\cdots\end{array}$ & $\begin{array}{l}0.40(35.6)^{1} \\
0.36(32.0)\end{array}$ & $\begin{array}{l}0.37(32.9)^{1} \\
0.51(45.4)\end{array}$ & $\begin{array}{c}0.66(58 \cdot 7)^{1} \\
-\end{array}$ & $\begin{array}{l}2 \cdot 66(237)^{1} \\
2 \cdot 74(244)\end{array}$ & $\begin{array}{l}4 \cdot 20(374)^{1} \\
5 \cdot 23(465)\end{array}$ & $\begin{array}{l}5 \cdot 20(463)^{1} \\
5 \cdot 61(499)\end{array}$ \\
\hline $\begin{array}{l}\text { Standard de } \\
\text { Venous } \\
\text { Capillary }\end{array}$ & $\begin{array}{l}\text { iati } \\
\ldots \\
\ldots\end{array}$ & $\begin{array}{l}\ldots \\
\ldots\end{array}$ & $\begin{array}{l}0.25(22 \cdot 2) \\
0.19(16.9)\end{array}$ & $\begin{array}{l}0.19(16.9) \\
0.34(30.2)\end{array}$ & $\begin{array}{c}0.55(48.9) \\
-\end{array}$ & $\begin{array}{l}2 \cdot 37(211) \\
1 \cdot 81(161)\end{array}$ & $\begin{array}{l}2 \cdot 38(212) \\
3 \cdot 55(316)\end{array}$ & $\begin{array}{l}1.94(173) \\
1.89(168)\end{array}$ \\
\hline $\begin{array}{l}\text { Range } \\
\text { Venous } \\
\text { Capillary }\end{array}$ & $\begin{array}{l}\cdots \\
\cdots\end{array}$ & . & $\begin{array}{c}0 \cdot 2-0 \cdot 8 \\
(17 \cdot 8-71 \cdot 2) \\
0 \cdot 2-0 \cdot 6 \\
(17 \cdot 8-53 \cdot 4)\end{array}$ & $\begin{array}{c}0 \cdot 2-0 \cdot 9 \\
(17 \cdot 8-80 \cdot 1) \\
0 \cdot 2-1 \cdot 4 \\
(17 \cdot 8-125)\end{array}$ & $\begin{array}{c}0 \cdot 03-2 \cdot 3 \\
(2 \cdot 7-205) \\
-\end{array}$ & $\begin{array}{c}0 \cdot 5-10 \cdot 3 \\
(44 \cdot 5-916) \\
1 \cdot 1-8 \cdot 5 \\
(97 \cdot 9-756)\end{array}$ & $\begin{array}{c}1 \cdot 1-8 \cdot 9 \\
(97 \cdot 9-792) \\
1 \cdot 5-13 \cdot 3 \\
(133-1183)\end{array}$ & $\begin{array}{c}2 \cdot 2-7 \cdot 8 \\
(196-694) \\
1 \cdot 9-7 \cdot 7 \\
(169-685)\end{array}$ \\
\hline
\end{tabular}

${ }^{1}$ Corresponding SI units $(\mathrm{nmol} / \mathrm{l})$ in parentheses

these men had been exposed for five to eight weeks prior to examination. Results of $\mathrm{CdB}$ done on venous and capillary specimens are summarized in Table 6. Although the low blood findings confirmed the absence of recent exposure, the urinary results showed quantities ranging from 17.8 to $738 \mathrm{nmol} / \mathrm{l}$ $(2-83 \mu \mathrm{g} / \mathrm{l})$ in nine of the 33 workers. It was not detectable in the remaining 24 . It is well known that urinary excretion of cadmium persists long after exposure to it has ceased.

Workers engaged in the manufacture of batteries Venous blood from 130 men and women making nickel-cadmium dry batteries was examined (Table 6). These workers handle plates impregnated with cadmium under ventilated hoods. Regular cadmium

TABLE 7

Findings in Pigment Factory Workers

\begin{tabular}{|c|c|c|c|c|c|c|c|}
\hline & \multicolumn{4}{|c|}{$\begin{array}{c}\mu g C d / 100 \mathrm{ml} \text { blood } \\
P D\end{array}$} & \multicolumn{3}{|c|}{$\begin{array}{c}\mu g C d / l \text { urine } \\
\text { (not corrected for } S G)\end{array}$} \\
\hline & \multicolumn{2}{|c|}{ Venous } & \multicolumn{2}{|c|}{ Capillary } & \multicolumn{2}{|c|}{ POL } & $S G$ \\
\hline $\begin{array}{r}1 \\
2 \\
3 \\
4 \\
5 \\
6 \\
7 \\
8 \\
9 \\
10 \\
11 \\
12 \\
13 \\
14\end{array}$ & $\begin{array}{r}0.5 \\
1.0 \\
1.5 \\
1.7 \\
1.7 \\
1.8 \\
1.8 \\
2.0 \\
2.0 \\
2.8 \\
3.0 \\
3.3 \\
3.8 \\
10.3\end{array}$ & $\begin{array}{l}(44 \cdot 5)^{1} \\
(89) \\
(133) \\
(151) \\
(151) \\
(160) \\
(160) \\
(178) \\
(178) \\
(249) \\
(267) \\
(294) \\
(338) \\
(916)\end{array}$ & $\begin{array}{l}1 \cdot 7 \\
1 \cdot 1 \\
1 \cdot 4 \\
2 \cdot 6 \\
1 \cdot 3 \\
2 \cdot 6 \\
3 \cdot 0 \\
2 \cdot 4 \\
2 \cdot 4 \\
2 \cdot 8 \\
2 \cdot 6 \\
2 \cdot 2 \\
3 \cdot 7 \\
8 \cdot 5\end{array}$ & $\begin{array}{l}(151)^{1} \\
(97 \cdot 9) \\
(125) \\
(231) \\
(116) \\
(231) \\
(267) \\
(214) \\
(214) \\
(249) \\
(231) \\
(196) \\
(329) \\
(756)\end{array}$ & $\begin{array}{c}\text { ND } \\
\text { ND } \\
\text { ND } \\
\text { ND } \\
4 \cdot 6 \\
\text { ND } \\
3 \cdot 0 \\
\text { ND } \\
3 \cdot 9 \\
\text { ND } \\
\text { ND } \\
7 \cdot 2 \\
13 \cdot 8 \\
47 \cdot 0\end{array}$ & $\begin{array}{l}(40 \cdot 9)^{1} \\
(26 \cdot 7) \\
(34 \cdot 7)\end{array}$ & $\begin{array}{l}1001 \\
1018 \\
1011 \\
1020 \\
1020 \\
1005 \\
1006 \\
1018 \\
1018 \\
1024 \\
1008 \\
1017 \\
1014 \\
1020\end{array}$ \\
\hline
\end{tabular}

${ }^{1}$ Corresponding SI units (nmol/l) in parentheses $\mathrm{ND}=$ not detectable 
TABLE 8

Findings IN SMelting Factory Workers

\begin{tabular}{|c|c|c|c|c|c|c|c|}
\hline & \multicolumn{4}{|c|}{$\begin{array}{c}\mu g C d / 100 \mathrm{ml} \text { blood } \\
P D\end{array}$} & \multicolumn{3}{|c|}{$\begin{array}{c}\mu g C d / l \text { urine } \\
\text { (not corrected for } S G \text { ) }\end{array}$} \\
\hline & \multicolumn{2}{|c|}{ Venous } & \multicolumn{2}{|c|}{ Capillary } & \multicolumn{2}{|c|}{$P O L$} & $S G$ \\
\hline $\begin{array}{l}1 \\
2 \\
3 \\
4 \\
5 \\
6 \\
7 \\
8\end{array}$ & $\begin{array}{l}7 \cdot 8 \\
4 \cdot 8 \\
2 \cdot 2 \\
2 \cdot 8 \\
6 \cdot 8 \\
5 \cdot 7 \\
6 \cdot 5 \\
5 \cdot 0\end{array}$ & $\begin{array}{l}(694)^{1} \\
(427) \\
(196) \\
(249) \\
(605) \\
(507) \\
(578) \\
(445)\end{array}$ & $\begin{array}{l}7 \cdot 7 \\
5 \cdot 5 \\
1 \cdot 9 \\
5 \cdot 4 \\
7 \cdot 2 \\
5 \cdot 0 \\
7 \cdot 4 \\
4 \cdot 8\end{array}$ & $\begin{array}{l}(685)^{1} \\
(489) \\
(169) \\
(480) \\
(641) \\
(445) \\
(658) \\
(427)\end{array}$ & $\begin{array}{r}35 \cdot 0 \\
14 \cdot 8 \\
12.6 \\
9.7 \\
75 \cdot 0 \\
31 \cdot 0 \\
22.0 \\
31.0\end{array}$ & $\begin{array}{c}(311)^{1} \\
(132) \\
(112) \\
(86 \cdot 3) \\
(667) \\
(276) \\
(196) \\
(276)\end{array}$ & $\begin{array}{l}1018 \\
1024 \\
1022 \\
1026 \\
1023 \\
1019 \\
1022 \\
1014\end{array}$ \\
\hline
\end{tabular}

${ }^{1}$ Corresponding SI units $(\mathrm{nmol} / \mathrm{l})$ in parentheses

in air measurements were done and usually found to be well below the current threshold limit value (TLV) for cadmium dust $\left(0.2 \mathrm{mg} / \mathrm{m}^{3}\right)$. Results of $\mathrm{CdB}$ ranged from $2 \cdot 70$ to $205 \mathrm{nmol} / 1(0 \cdot 03-2 \cdot 30 \mu \mathrm{g} / 100 \mathrm{ml})$, the mean being $58.7(0.66)$. Urines were also examined by POL but cadmium was detected in only four out of 95 urines and then in low quantity only, the highest being $117 \mathrm{nmol} / \mathrm{l}(13 \cdot 1 \mu \mathrm{g} / \mathrm{l})$. Detailed results are not given but the findings are summarized in Table 9.

Workers exposed to cadmium pigments

Detailed results using capillary and venous blood, and the urinary findings in 14 workers engaged in pigment manufacture, are given in Table 7 and summarized in Table 6. Environmental measurements had not been taken recently in this factory. We also sampled managerial, clerical, and laboratory staff whose findings were normal and are excluded from the Table.

\section{Workers in two cadmium smelting factories}

Results in 25 workers in two factories exposed to cadmium fume are also summarized in Table 6 and the detailed findings in the second factory are given in Table 8. Environmental measurements in this factory showed seven samples out of 10 to be above the current TLV for cadmium fume $\left(0 \cdot 1 \mathrm{mg} \mathrm{Cd} / \mathrm{m}^{3}\right)$ Five workers in the first factory with only intermittent exposure are excluded from Table 6.

\section{Statistical analysis of results}

\section{Venous against capillary $\mathbf{C d B}$}

There is a good correlation ( 0.93$)$ between the results of $\mathrm{CdB}$ done on venous and capillary specimens (Fig. 3). All results are included and the mean of the venous samples was $155 \mathrm{nmol} / \mathrm{l}(\mathrm{SD}=202)(1.74$ $\mu \mathrm{g} / 100 \mathrm{ml}(\mathrm{SD}=2 \cdot 27))$ and the mean of the capillary samples was $180 \mathrm{nmol} / \mathrm{l}(\mathrm{SD}=238)(2.02 \mu \mathrm{g} / / 100$
$\mathrm{ml}(\mathrm{SD}=2 \cdot 67))$. Capillary results tend to be higher in the exposed groups which could be regarded as an advantage in the context for which the technique is recommended in that the method fails safe.

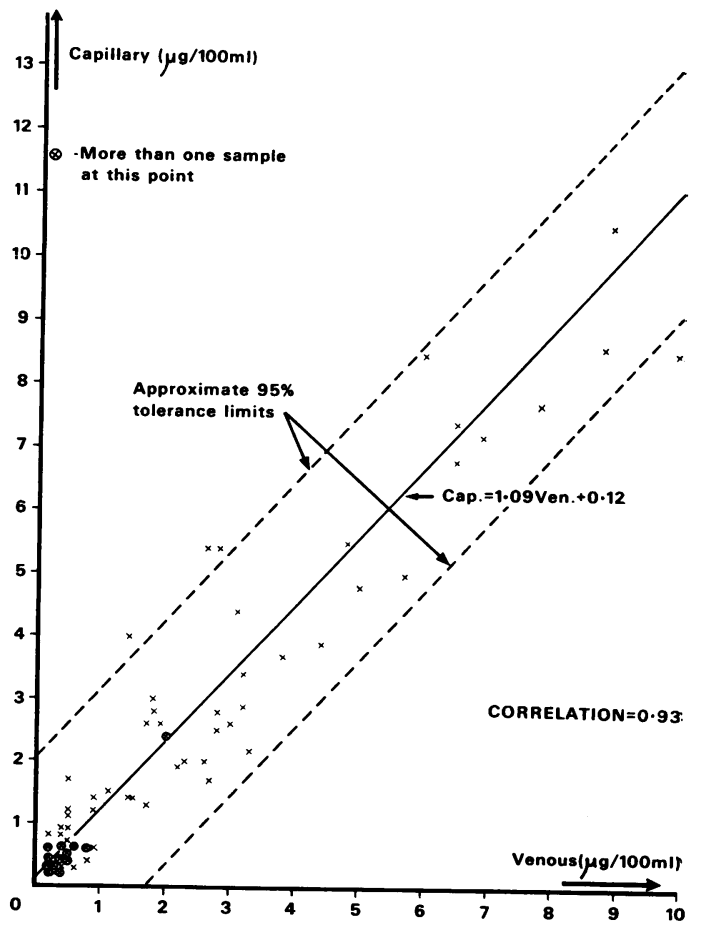

FIG. 3. A comparison of results using capillary and venous blood. The regression line is shown and the approximate $95 \%$ tolerance limits.

Conversion: Traditional Units to SI

-Cadmium: $1 \mu \mathrm{g} / 100 \mathrm{ml}=88.9 \mathrm{nmol} / \mathrm{l}$. 
TABLE 9

Comparison of Blood and Urine CADMIUM

\begin{tabular}{|c|c|c|c|c|c|c|c|c|c|c|c|c|}
\hline & \multicolumn{12}{|c|}{$C d$ in urine $(\mu g / l)$} \\
\hline & \multicolumn{3}{|c|}{ Not detectable } & \multicolumn{3}{|c|}{$\leq 5(44 \cdot 5)^{1}$} & \multicolumn{3}{|c|}{$\begin{array}{l}\geq 5(44 \cdot 5)^{1} \\
\leq 10(89)\end{array}$} & \multicolumn{3}{|c|}{$>10(89)^{1}$} \\
\hline & No. & Mean & $S D$ & No. & Mean & $S D$ & No. & Mean & $S D$ & No. & Mean & $S D$ \\
\hline $\begin{array}{l}\text { Cd in blood } \\
(\mu \mathrm{g} / 100 \mathrm{ml})\end{array}$ & 134 & $\begin{array}{r}0.64 \\
(56.9)\end{array}$ & $\begin{array}{r}0.54 \\
(48 \cdot 0)\end{array}$ & 22 & $\begin{array}{r}1.91 \\
(170)\end{array}$ & $\begin{array}{r}2 \cdot 24 \\
(199)\end{array}$ & 6 & $\begin{array}{r}2 \cdot 43 \\
(216)\end{array}$ & $\begin{array}{r}1.53 \\
(136)\end{array}$ & 24 & $\begin{array}{r}3.63 \\
(323)\end{array}$ & $\begin{array}{r}3.00 \\
(267)\end{array}$ \\
\hline
\end{tabular}

${ }^{1}$ Corresponding SI units (nmol/l) in parentheses

Comparison of blood and urinary cadmium

Comparisons of $\mathrm{CdB}$ and $\mathrm{CdU}$ show that if cadmium is detected in the urine then the mean $\mathrm{CdB}$ of the workers concerned is greater than the corresponding mean in those with no cadmium detectable in the urine. There also appears to be an upward trend in the mean $\mathrm{CdB}$ as the amount of $\mathrm{CdU}$ increases (Table 9). However, the range of the $\mathrm{CdB}$ at the different urinary concentrations was wide.

\section{Discussion}

Flame atomic absorption methods do not satisfactorily separate volatile cadmium from a burning blood matrix unless the sample has previously been oxidized. Even so, Ediger and Coleman (1973) observed that 'An unusual amount of care was necessary to achieve accurate results'. With the flameless graphite cup technique no significant loss or retention of cadmium was seen during the controlled ashing step (Tables 1 and 2). Frequent monitoring of the non-atomic signal is essential to ensure that the ashing temperature does not vary. For the determination of the lowest concentrations of cadmium in the unexposed, however, continuous background correction is essential, since the nonatomic absorption element of the total cadmium signal and the possible variation of the ash control could result in imprecision and unacceptable loss at these levels. SBWs of approximately 0.15-0.99 nm used in conjunction with atomization temperatures above $600^{\circ} \mathrm{C}$ resulted in curved calibration graphs over the range $17 \cdot 8-890 \mathrm{nmol} \mathrm{Cd} / 1$ blood $(0 \cdot 2-10 \mu \mathrm{g} /$ $100 \mathrm{ml}$ ). This was considered to be due to an inadequate amplifier response to the speed of atomization, especially at cadmium concentrations above $534 \mathrm{nmol} / \mathrm{l}(6 \mu \mathrm{g} / 100 \mathrm{ml})$. When the atomization temperature was set at $600^{\circ} \mathrm{C}$ with SBWs wider than $0.33 \mathrm{~nm}$, curvalure persisted. The analytical line invariably curved towards the concentration axis and approached an asymptote at a limiting absorbance. This suggested that the problem was due to residual unabsorbed light, correctable by the use of an optimum SBW (Fig. 2, graph A). The validity of the line was confirmed by checking the value of a control blood whose concentration was known (Fig. 2). The second non-atomic signal occurred at a constant measurable time after the cadmium signal and therefore did not interfere.

Although the CdB does not necessarily reflect the body burden it would seem from the results reported here that it may well prove a valuable index of the factory hazard (Table 6).

Until recently results of cadmium determinations in blood at the levels found in unexposed workers have been conflicting. Willden (1973) found approximately $178 \mathrm{nmol} / \mathrm{l}(2 \mu \mathrm{g} / 100 \mathrm{ml})$ in normal subjects, while earlier reports, for example Butt et al. (1964), have quoted much greater quantities. Problems of methodology may well account for these variations. Cadmium quantities of less than $89 \mathrm{nmol} / \mathrm{l}(1 \mu \mathrm{g} / 100$ $\mathrm{ml}$ ) quoted by Friberg, Piscator, and Nordberg (1971) for unexposed workers in Sweden, the United States of America, and West Germany are of the same order as reported here.

There is general agreement that, despite fluctuations, $\mathrm{CdB}$ concentrations in exposed workers are significantly higher than in unexposed controls. Friberg et al (1971) and our experience certainly bear this out.

Although the published data (Friberg et al., 1971) suggest that the CdB may not prove a useful index for the control of exposure in man, further investigation is clearly needed. The method described here may facilitate this.

We are indebted to Mr. P. F. Collier who was responsible for the statistical analyses.

\section{References}

Butt, E. M., Nusbaum, R. E., Gilmour, T. C., Didio, S. L., and Sister Mariano (1964). Trace metal levels in human serum and blood. Archives of Environmental Health, 8, 52-57.

Cernik, A. A. (1974). The determination of blood lead using a $4.0 \mathrm{~mm}$ paper punched disc carbon sampling 
cup technique. British Journal of Industrial Medicine, 31, 239-244.

and Sayers, M. H. P. (1971). Determination of lead in capillary blood using a paper punched disc atomic absorption technique. British Journal of Industrial Medicine, 28, 392-398.

Dolinsek, F., Stupar, J., and Glazer, L. (1972). Determination of $\mathrm{Pb}$ and $\mathrm{Cd}$ in whole blood by flameless atomic absorption technique. 4th Yugoslav Conference on Applied Spectroscopy, pp. 217-221. Union of Yugoslav Chemical Society, Zagreb 2-4 October. Institut 'Boris Kidrie', Hajdrihova 29, 61000 Ljubljana, Jugoslavia.

Ediger, R. D. and Coleman, R. L. (1973). Determination of cadmium in blood by a Delves cup technique. Atomic Absorption Newsletter, 12, 3-6.

Friberg, L., Piscator, M., and Nordberg, G. (1971). Cadmium in the Environment, pp. 50, 63, 65. C.R.C. Press, Cleveland, Ohio, U.S.A.

Hauser, T. R., Hinners, T. A., and Kent, J. L. (1972). Atomic absorption determination of cadmium and lead in whole blood by a reagent-free method. Analytical Chemistry, 44, 1819-1821.
Joselow, M. M. and Bogden, J. D. (1972). Multi element microanalysis by Delves cup A.A. spectrophotometry chelate/solvent extracts. Atomic Absorption Newsletter, 11, 127-128.

Lieberman, K. W. (1973). Determination of cadmium in biological fluids by the Delves modification of atomic absorption spectrophotometry. Clinical Chimica Acta, 46, 217-221.

Rainsford, S. G. (1968). The problem of the medical supervision of lead workers. Journal Royal Naval Medical Services, 54, 69-79.

Ullucci, P. A. and Hwang, J. Y. (1973). Determination of Cadmium in Biological Materials by Flame and Non-flame Atomic Absorption Techniques, pp. 563-567. Applications Laboratory, Instrumentation Laboratory Inc., 113 Hartwell Avenue, Lexington, Mass.

Willden, Elizabeth G. (1973). Urinary and whole blood cadmium concentrations in renal disease. Annals of Clinical Biochemistry, 10, 107-110.

Received for publication 12 July 1974.

Accepted for publication 1 August 1974. 